\title{
Vibration and Modal Analysis of Small Induction Motor
}

\author{
Yan $\mathrm{LI}^{1,}$, , Jianmin DU ${ }^{1, \mathrm{~b}}$, Jiakuan XIA ${ }^{1}$ \\ ${ }^{1}$ Research Institute of Special Electrical Machines, Shenyang University of Technology, Shenyang, \\ 110870, China \\ aemail: liyanty@yahoo.com, bemail: jianmin2015_sut@163.com
}

Keywords: Induction motor; Finite element; Natural frequency; hammering method

\begin{abstract}
The vibration and noise of the motor is a significant factor to evaluate the quality of the motor and the modal analysis is one of the main approaches to study the structural dynamic characteristics of motor. The motor will be in the resonance state when the excitation frequency of the harmonic electromagnetic force is close or consistent to the natural frequency of the motor, thus produce vibration and noise with high amplitude. Aim at the modal analysis problem of motor, firstly the three-dimensional (3-D) whole induction motor model including components, e.g., stator, frame, cover, is elaborately established using Pro/Engineer software; then the model problem of motor is simulated and analyzed based on finite element method (FEM), and compute and analyze the relevant components for the effect; of motor structural modal respectively; finally, compare the computation results with the experimental ones to verify the correctness of the proposed approach through the hammering method test. Through the computation and analysis of the motor model in this paper, some basis references and foundation have been established for the design and further research of motor.
\end{abstract}

\section{Introduction}

The electrical motors are used in different industrial applications and they have to run continuously thought their operating regime. With the development of the motor industry and technology, vibration and noise of the motor has become an important indicator to evaluate the quality of motor [1]. The motor will be in the resonance state when the excitation frequency of the harmonic electromagnetic force is close or consistent to the natural frequency of the motor, thus produce vibration and noise with high amplitude [2] [3]. In order to reduce the vibration and noise of the motor, it is necessary to start from the generation mechanism that accurately determine the excitation frequency of the motor, Therefore, the study of motor parts and modal natural frequency is very important[4].

Usually, it mainly analyzes the influence of motor stator and frame on natural frequency [5] [6]. The theoretical and experimental modal analysis highlights the influence of the complex structure on natural frequencies. In many papers, based on the experience, the modal study was extended to the whole three-phase squirrel-cage induction motor [7] [8]. However, the factors, such as rotor, cover and others, should also be considered comprehensively in modal analysis and calculation. At the same time, how to establish an accurate simulation model is also the key point in the analysis process.

In this paper, it is focused on analyzing the impact of motor parts on the motor mode. Finally, compare the computation results with the experimental ones to verify the correctness of the proposed approach through the hammering method test.

\section{Modal analysis of mathematical model}

In general, based on vibration theory, analysis method to modal parameters for the target is called modal analysis. In other words, modal analysis is the study of relationship between the physical parameters of system model, modal parameter model and non-parameter model.

The vibrating structure can be discretized into finite degree of freedom system. For one vibration system with $\mathrm{n}$ degrees, it is required $\mathrm{n}$ separate physical coordinates to describe the physical 
parameters of the model.

According to the principle of Hamilton, the relationship of stress to strain and strain to displacement, the Lagrange equation of motion is

\section{$\mathbf{K u}+R \dot{u}+M \ddot{u}=F$}

In the type, $\boldsymbol{K}$ is the stiffness matrix; $\boldsymbol{R}$ is the damping matrix; $\boldsymbol{M}$ is the mass matrix, $\boldsymbol{u}$ is the displacement vector of node, and point superscript expressed the differential of the time; $\boldsymbol{F}$ is the external incentive nodal force vector.

Because the calculation of natural frequency of the motor is generally undamped free vibration, so make $\boldsymbol{F}=\mathbf{0}$ in (1) and the special solution can be written:

$\boldsymbol{u}=\boldsymbol{\Phi} \boldsymbol{e}^{j \omega t}$

In the type (2), $\boldsymbol{\phi}$ is the free response amplitude array.

Put the type (2) into the type (1), the type (3) can be obtained:

$\left(\boldsymbol{K}-\omega^{2} \boldsymbol{M}\right) \boldsymbol{\Phi}=\mathbf{0}$

This equation has the necessary and sufficient conditions for the zero solution is the coefficient matrix determinant equal to zero, that is:

$\left|\boldsymbol{K}-\omega^{2} \boldsymbol{M}\right|=0$

The solution of the above equation can be obtained natural frequencies and mode shapes.

\section{The sample motor and finite element calculation model}

This paper is mainly about the finite element modal analysis and calculation of a small induction motor, and the technical parameters of the sample motor structure are shown in Table 1.

Tab. 1 Technical parameters of the sample motor structure

\begin{tabular}{cc}
\hline Parameter & Value \\
\hline Rated power & $7.5 \mathrm{~kW}$ \\
Rated speed r/min & 1000 \\
The core length of stator and rotor $/ \mathrm{mm}$ & 140 \\
The slots of stator and rotor & $36 / 28$ \\
\hline
\end{tabular}

This paper uses 3-D modeling software Pro/Engineer to establish the stator, frame, cover and rotor according to the actual size of the motor.

Due to the actual model of the motor is relatively complex; the meshing work becomes very large, which may impact on the accuracy of the final calculation. In order to shorten the time of finite element calculation process, to ensure the rationality of meshing the model, and improve the accuracy of calculation, therefore the motor model should be simplified in a certain extent. The 3-D models of the motor are shown in Figure 1.

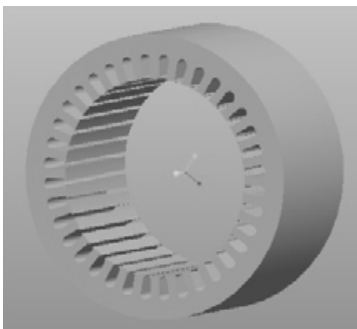

(a) Single stator

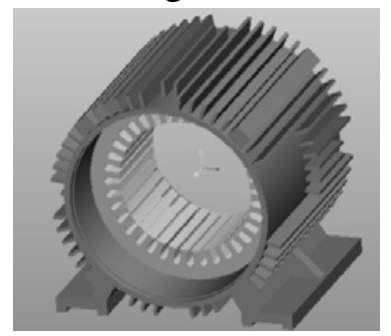

(b) Stator and frame

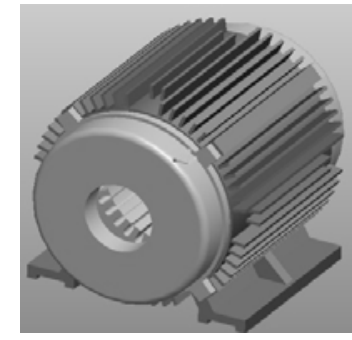

(c) Stator, frame and cover

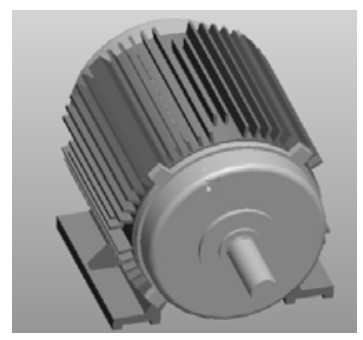

(d) Whole motor

Fig.1. Three-dimensional structural model of the motor

One of most important factors in the modal analysis and calculation is the reasonable selection in material properties. Due to the structure of the stator and rotor is composed of a certain thickness of the silicon steel, so considering the anisotropic materials is needed in the process of calculation. Based on reference [9], elastic modulus perpendicular to the direction of the stator laminations is generally lower than the elastic modulus in the plane of the laminated stator core, and the density of laminated core material is lower than solid steel of the same volume density. Therefore, the corresponding material properties should be clear in the process of calculation [10]. The Material 
parameters of motor are shown in the Table 2.

Tab. 2 Material parameters of motor modal analysis

\begin{tabular}{cccc}
\hline Structural components & Modulus of elasticity/GPa & Poisson's ratio & Density $\left(\mathrm{kg} / \mathrm{m}^{3}\right)$ \\
\hline Stator and rotor & Ez $=205$ & 0.3 & 7400 \\
Cover and frame & 113 & 0.23 & 7200 \\
Bar & 7 & 0.32 & 2700 \\
shaft & 205 & 0.3 & 7800 \\
\hline
\end{tabular}

In the whole calculation process, As far as possible choose hexahedron mesh is chosen as far as possible in order to get more optimal solution of the calculation accuracy and faster speed. The modal frequency results are shown in Table 3.

Tab. 3 Modal frequency results for the sample motor

\begin{tabular}{ccccc}
\hline Order & 2 & 3 & 4 & 5 \\
\hline Modal a & 717.17 & 1945.3 & 3534.8 & 5346.2 \\
Modal b & 735.89 & 1974.5 & 2739.8 & 3312.9 \\
Modal c & 967.17 & 2111.3 & 3057.7 & -- \\
Modal d & 1344.2 & 2011.9 & 2710.2 & 3352.3 \\
\hline
\end{tabular}

From the Tab.3, the motor model in the 2nd order modal frequency is relatively low, which is easy to achieve the resonance state. FEM results of mode shapes for the motor stator and cover are shown in Figure 2 and Figure 3.

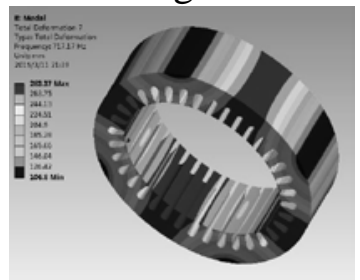

(a)The 2nd mode shape

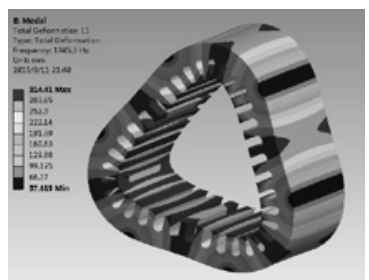

(b) The 3rd mode shape

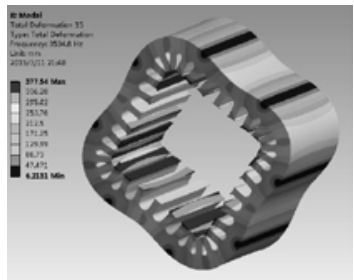

(c)The 4th mode shape

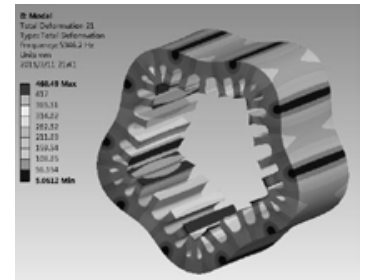

(d)The 5th mode shape

Fig. 2 FEM results of mode shapes for the motor stator

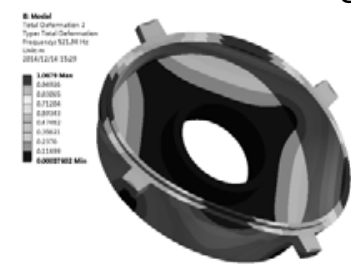

(a)The 2nd mode shape

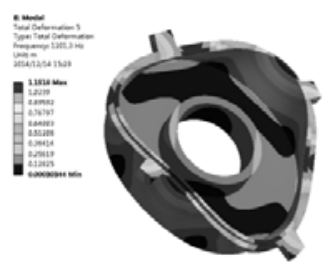

(b) The 3rd mode shape

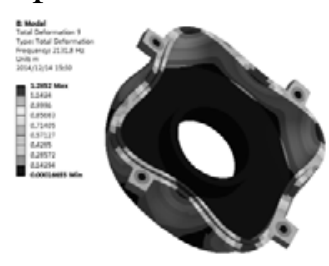

(c) The 4th mode shape

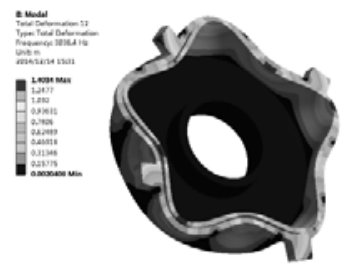

(d) The 5th mode shape

Fig. 3 FEM results of mode shapes for the motor and cover

As can be seen from Figure 2 and Figure3, the stator and cover of the motor can impact the modal shape and natural frequency in a certain degree; the rotor of the motor, which increase the stiffness of the motor, also impact the shape and natural frequency in a certain degree. All in all, in order to predict the natural frequency of the motor structure accurately, it is necessary to establish the complete FEM structure of the motor.

\section{The hammering modal test of the sample motor}

To test and verify the accuracy of the model of finite element simulation results, BVM-300-4M Four-channel vibration test instrument is used in this paper. The instrument is shown in Figure 4.

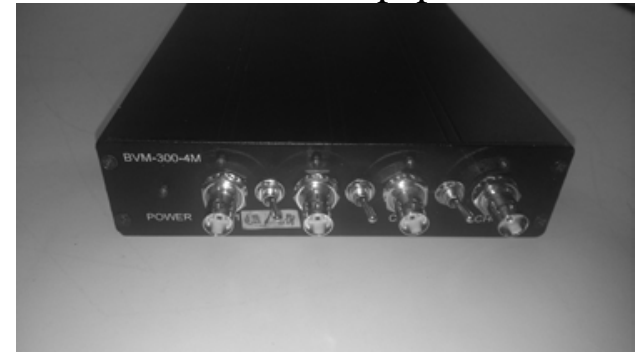

Fig. 4 BVM-300-4M Four-channel vibration test instrument 
Frequency domain method of modal analysis is the common method to identify the model parameters for the test data of known excitation and response. During the process of collecting data, the excitation and response signal need to collect at the same time. The whole process of modal test is shown in Figure 5.

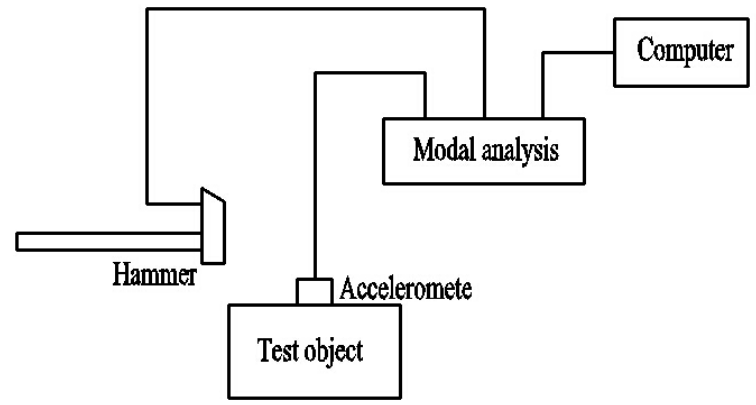

Fig. 5 Modal test sketch of sample motor

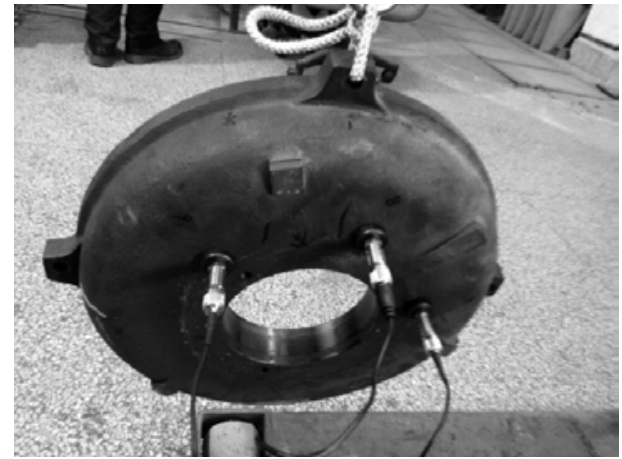

Fig. 6 Picture of sample motor cover

To agree with the process of finite element simulation, the spring of meeting the stiffness requirement is used to simulate the free state of the motor parts. The experiment of the motor cover is shown in Figure 6.

\section{Test results}

By the hammering method, the excitation signal and the corresponding response signal can be obtained in the process of the experiment. They are shown in Figure 7.

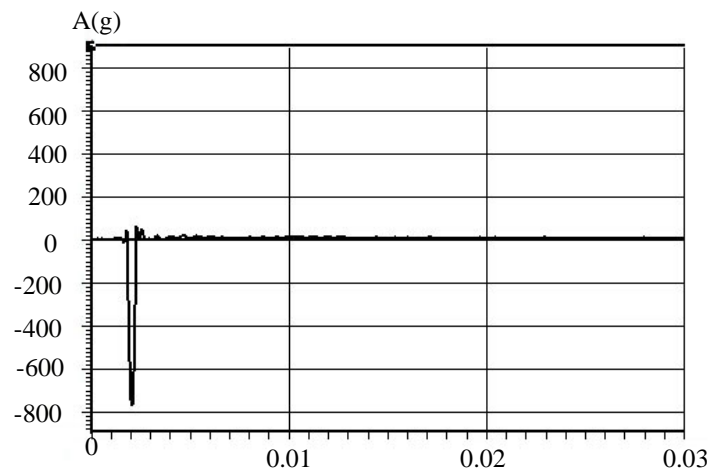

(a) The excitation signal of the motor cover

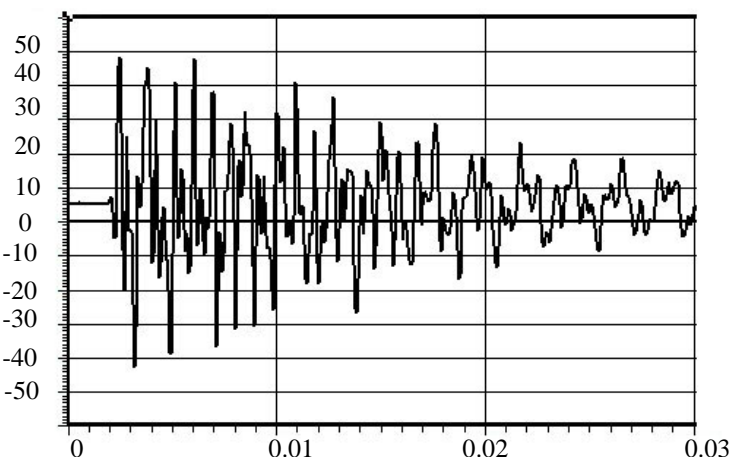

(b) The response signal of the motor cover

Fig. 7 The excitation signal and the response signal of the motor cover

According to the excitation signal and the response signal above, the natural frequency of the sample motor test results can be obtained as shown in Table 4.

Tab. 4 Comparison of experimental and simulation results

\begin{tabular}{ccccc}
\hline Modal order & 2 & 3 & 4 & 5 \\
\hline FEM results $/ \mathrm{Hz}$ & 521.94 & 1201.3 & 2131.8 & 3036.4 \\
\hline Experiment results/Hz & 512 & 1216 & 2192 & 3104 \\
\hline Percentage deviation & $-1.9 \%$ & $1.2 \%$ & $3.8 \%$ & $2.2 \%$ \\
\hline
\end{tabular}

As can be seen form the table above, in addition to the test results of 2nd order slightly smaller than the simulation results, the rest of the other order experimental results are larger than the simulation results, the biggest percentage relative deviation is $3.8 \%$, which meets the requirements of engineering precision. It is shown that the FEM simulation results of the motor cover is consistent with the experimental results, thus it is verified the simplified method of this model in a certain extent.

\section{Conclusion}

In this paper, an induction motor is taken as the research object, the 3D model of each part of the motor structure is established, and modal analysis was carried out on the motor, at the same time, 
the influence of the motor cover, frame and the rotor to the natural frequency and mode is the key analysis. In order to compare the computation results with the experimental ones to verify the correctness of the proposed approach, the hammering method test is used. Because the simulation results of the motor cover is consistent with the experimental results, so the accuracy of the calculation method is verified, and the feasibility of the motor model simplification method is also verified.

\section{Acknowledgement}

In this paper, the research was sponsored by Program for LNIRT in university (LT2013006).

\section{References}

[1] Zhang Lei, Wen Xu-hui. Radial electromagnetic vibration model characteristics of PMSMs for electric vehicles [J]. Electric Machines and Control, 2012:16(5): 33-39.

[2] Tang Ren-yuan, Song Zhi-huan, Yu Shen-bo. Study on source of vibration and acoustic noise of permanent magnet machines by inverter [J].Electric Machines and Control, 2011:14(3): 12-17.

[3] BOESING Matthias, W de Doncker. Exploring a vibration synthesis process for the acoustic characterization of electric drives [J], EEEE Transactions on Industry Applications, 2012:48(1): 70-78.

[4]Li Xiao-hua, Huang Su-rong, LI Liang-zi. Calculation and analysis of vehicle vibration and noise of permanent magnet synchronous motor applied in electric vehicle [J].Electric Machines and Control, 2013:17( 8 ) : 37-42.

[4] S.A. Long, Z.Q. Zhu, D.Howe. Vibration behavior of stators of switched reluctance motors. IEE Proceedings, Electric Power Applications. 2001:148(3):257-264.

[5] Dai Ying, Cui Shu-mei, Song Li-wei. Finite Element Method Modal Analysis of Driving Motor for Electric Vehicle.[J]. Proceedings of the CSEE，2011:31(9): 100-104

[6] Zhou Ting. The Natural Frequces Computation of the Motor Stator Considering the Stator Rind Affection [J]. Modern motor, 2009, 33(2):33-39

[7] Fuminori Ishibashi, Makoto Matsushita, Shinichi Noda. Change of mechanical natural frequencies of induction motor. IEEE Transactions On Industry Applications.2010, 46(3):922-927

[8] Han Wei, Jia Qi-fen, QIU Jia-jun. Vibration and modal analysis for stator of an induction motor [J]. Journal of Vibration and Shock, 2012:33(17): 91-94.

[9] Wang Tian-yu, Wang Feng-xiang. Vibration and modal analysis of stator of large induction motors.[J]. Proceedings of the CSEE, 2007:27(12): 41-45.

[10] Huang Jie, Ji Zhong, Duan Hu-ming. Experimental modal analysis of mechanical structure and typical applications [J].China Measurement \& Test, 2010:36(2):4-8 